\title{
ARTÍGULO ORIGINAL: Frecuencia de trom- boembolismo pulmonar en pacientes con cáncer de páncreas en el periodo de enero 2009 a diciem- bre 2013 en el Hospital San Juan de Dios
}
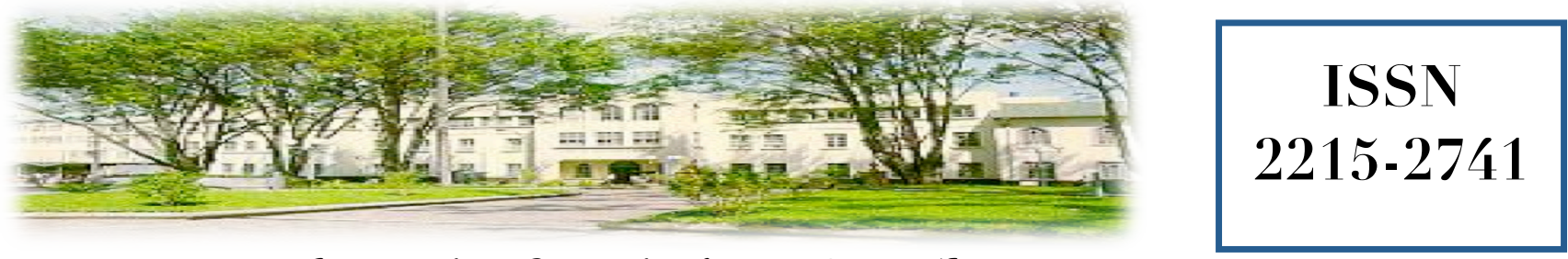

Hospital San quan de Dias, San José, Costa Rica. Fundado en 1845

Recibido: $\quad 08 / 03 / 2015$

Aceptado: $\quad 25 / 03 / 2015$

Carlos Fernando Estrada-Garzona ${ }^{1}$

Andrés Francisco Garzona-Navas ${ }^{2}$

\footnotetext{
${ }^{1}$ Médico-Cirujano. Profesor del Departamento de Farmacología. Escuela de Medicina. Universidad de Costa Rica. Correo electrónico: drestradag@gmail.com

${ }^{2}$ Médico-Cirujano. Universidad de Costa Rica. Clínica Quirúrgica la Inmaculada. Correo electónico: andresgarzona@gmail.com
}

\section{RESUMEN}

Antecedentes: La identificación de la frecuencia de tromboembolismo pulmonar en el paciente con cáncer de páncreas como factor de riesgo permite optimizar la sospecha, diagnóstico y prevención de una complicación vascular potencialmente fatal. Métodos: Estudio observacional descriptivo que incluyó 92 pacientes con diagnóstico de cáncer de páncreas en el periodo de enero 2009 a diciembre 2013 que se identificaron según la codificación del CIE-10 en la base de datos de egreso hospitalario de Registros Médicos y Estadística de Salud (REMES) del Hospital San Juan de Dios de Costa Rica. Resultados: En la muestra la edad promedio fue de 63.67 años, la relación sexo femenino: masculino de 1.24 (55.4\% mujeres, $44.6 \%$ hombres) y hubo un $14.1 \%$ de pacientes fallecidos. Los factores de riesgo para tromboembolismo pulmonar más frecuentes fueron el tabaquismo (31.8 \%), la cirugía abdominal $(29.3 \%)$ y la presencia de infección (14.1\%). Se identificó la presencia de tromboembolismo pulmonar en 2 pacientes (2.2\%), diagnosticados con tomografía computarizada con angiografía espiral (CTPA) y con defectos de perfusión proximales en ambos casos. Conclusiones: En los pacientes hospitalizados con diagnóstico de cáncer de páncreas los factores de riesgo para tromboembolismo pulmonar más frecuentes fueron el tabaquismo, la cirugía abdominal y la presencia de infección. 


\section{PALABRAS CLAVE}

Cáncer de páncreas, tromboembolismo pulmonar.

\begin{abstract}
Background: The knowledge of pulmonary embolism frequency in patients with pancreatic cancer as a risk factor allows the clinician to optimize suspicion, diagnosis and prevention of a potentially fatal vascular complication. Methods: Descriptive observational study that included 92 patients with a diagnosis of pancreatic cancer in the period of time from January 2009 to December 2013 that were identified according to CIE-10 codification in the database for hospitalization Medical Records and Health Statistics (REMES) of the San Juan de Dios Hospital, Costa Rica. Results: In the sample, mean age was 63.67 years, the relation female to male was 1.24 (55.4\% female, $44.6 \%$ male), and $14.1 \%$ of patients died. The most frequently found risk factors for pulmonary embolism were smoking (31.8\%), abdominal surgery (29.3\%) and the presence of infection (14.1\%). There were 2 patients diagnosed with pulmonary embolism $(2.2 \%)$ with the aid of spiral computed tomography angiography (CTPA) and in both cases proximal perfusion defects were identified. Conclusions: In patients hospitalized with a diagnosis of pancreatic cancer the most frequent risk factors for pulmonary embolism were smoking, abdominal surgery and the presence of infection.
\end{abstract}

\section{KEY WORDS}

Panceratic cancer, pulmonary embolism.

\section{INTRODUCCIÓN}

El tromboembolismo pulmonar (TEP) es evento clínico multicausal $^{(1,2)}$, responsable de gran morbilidad y mortalidad a nivel mundial ${ }^{(3-8)}$. Su incidencia anual es distinta entre pacientes ambulatorios y hospitalizados. En este último grupo se asocia con un aumento en la mortalidad al presentarse en pacientes de riesgo ${ }^{(9-16)}$.

El TEP posee diversos factores de riesgo como son los procesos infecciosos ${ }^{(17)}$, enfermedades inflamatorias $^{(18-22)}$, cirugía $^{(23,24)}$ cáncer, entre otros $^{(1,25,26)}$. Entre las principales neoplasias que presentan mayor incidencia anual de TEP figura el cáncer de páncreas ${ }^{(27)}$.

Este estudio pretende identificar la frecuencia de TEP en la población estudiada en aras de conocer el perfil epidemiológico de esta complicación vascular en pacientes de riesgo a nivel local. De manera que el conocimiento de la frecuencia de complicaciones asociadas al cáncer de páncreas ofrezca una mejora en la atención del paciente con este diagnóstico .

\section{MATERIALES Y MÉTODOS}

El diseño del estudio es observacional descriptivo con la población definida por los pacientes adultos, mayores de 18 años, hospitalizados con el diagnóstico de cáncer de páncreas en el Hospital San Juan de Dios en el periodo de enero 2009 a diciembre 2013.

El diseño experimental implica que se trabajó con la totalidad de la población (número total de pacientes definido por los registros médicos del Servicio de Estadística y el Servicio de Archivo del Hospital San Juan de Dios que cumplan los criterios de inclusión en el periodo de estudio).

Se define como criterios de inclusión del estudio las siguientes condiciones:

1. Edad mayor a 18 años.

2. Diagnóstico (reciente o previo a la hospitalización y confirmado con estudio histopatológico) de cáncer de páncreas (CIE-10 C25) incluyendo todas las categorías del CIE-10:

- (C25.0) neoplasias malignas de la cabeza del páncreas.

- (C25.1) neoplasias malignas del cuerpo del páncreas.

- (C25.2) neoplasias malignas de la cola del páncreas.

- (C25.3) neoplasias malignas del conducto pancreático.

- (C25.4) neoplasias malignas del endocrino del páncreas.

- (C25.7) neoplasias malignas de otras partes del páncreas.

- (C25.8) neoplasias malignas del solape del páncreas.

- (C25.9) neoplasias malignas sin especificar del páncreas. 
3. Diagnóstico de cáncer de páncreas (confïmado con reporte histopatológico en el expediente).

Por otro lado, las siguientes condiciones se consideraron como criterios de exclusión del estudio:

1. Pacientes menores de 18 años.

2. Paciente con diagnóstico de TEP realizado con base en sospecha clínica, sin confirmación con tomografía computarizada con angiografía espiral (ANGIOTAC / CTPA) (reportado por radiólogo especialista) o gammagrafía ventilación perfusión (reportado por médico especialista en medicina nuclear).

Las variables de estudio establecidas para análisis estadístico son Cáncer de Páncreas Código CIE-10 (C25), tromboembolismo pulmonar Código CIE-10 (I126), condición de egreso, sexo, edad, peso, índice de masa corporal (IMC), tabaquismo, antecedente de trombosis venosa profunda (TVP) (diagnosticado con ultrasonido dúplex miembros inferiores), embarazo / periparto, enfermedad inflamatoria intestinal (CUCI, Chron), infección aguda, vasculitis, cirugía (ortopedia, abdomen, tórax, neurocirugía, urología, necesidad de transfusión (glóbulos rojos empacados, plaquetas, plasma fresco congelado, crioprecipitados), enfermedad pulmonar obstructiva crónica (EPOC) (Gold A, B, C, D), insuficiencia cardíaca congestiva (ICC) (NYHA I, II, III, IV), trastorno hematológico no neoplásico y enfermedad varicosa de miembros inferiores.

Además, se valoró como variables de estudio la presencia del defecto de perfusión (proximal, segmentario, sub-segmentario, periférico, unilateral, bilateral), biomarcadores de lesión miocárdica (troponina I, BNP), hipotensión, electrocardiograma anormal, ecocardiograma anormal, tomografía axial computarizada de tórax anormal.

Las variables anteriores se incluyeron en la Hoja de Recolección de Datos y fueron utilizados para establecer si el tromboembolismo pulmonar se clasifica como masivo, submasivo o de bajo riesgo.

El protocolo fue debidamente aprobado por el Comité Local de Bioética en Investigación (CLOBi). Para el análisis estadístico se utiliza el paquete IBM ${ }^{\circledR}$ SPSS Statistics 9.0, considerando que el diseño experimental permite análisis descriptivo de los resultados del estudio.

Dado que la muestra a analizar está determinada por la base de datos de registros médicos y los criterios de inclusión y/o exclusión, no se utiliza ninguna técnica de muestreo que garantice la selección aleatoria de la muestra dado el diseño experimental. Además, la reducción del número de pacientes evaluados por ausencia de información en el expediente para completar los criterios de inclusión (no reporte de estudios confirmatorios de TEP a pesar del diagnóstico de egreso) es una limitante del estudio contemplada antes de iniciar la recolección de datos. Cabe mencionar que se considera la presencia de variables confusoras como otros factores de riesgo para tromboembolismo pulmonar que pueden influir en los resultados de frecuencia de este evento en la población estudiada. Por esta razón, estas variables confusoras se contemplan como variables de estudio para determinar su frecuencia en la muestra y realizar un análisis objetivo de la información.

\section{RESULTADOS}

De un total de 114 pacientes con diagnóstico de cáncer de páncreas en el periodo de estudio, se incluyó para análisis estadístico a 92 pacientes que cumplieron con los criterios de exclusión e inclusión establecidos en el diseño experimental (Figura 1). Se identificó a un $14.1 \%$ de pacientes fallecidos en la muestra, la edad promedio de la misma fue de 63.67 años y la relación sexo femenino: masculino fue de 1.24 (55.4\% mujeres, $44.6 \%$ hombres) (Tabla I, Cuadro 1 y 2). Los factores de riesgo para tromboembolismo pulmonar más frecuentes fueron el tabaquismo $(31.8 \%)$, la cirugía abdominal $(29.3 \%)$ y la presencia de infección (14.1\%).

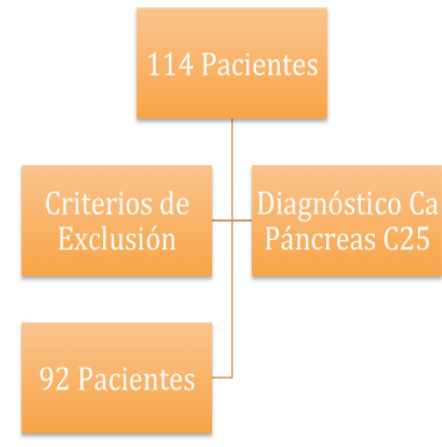

Figura 1: Selección de población de estudio con diagnóstico de Cáncer de Páncreas. 


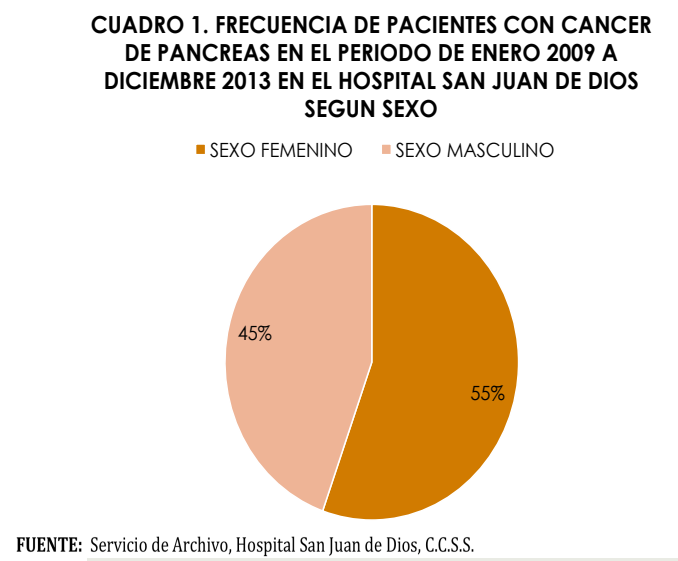

Tabla I. Características de pacientes con cáncer de páncreas en el periodo de enero 2009 a diciembre 2013 en el Hospital San Juan De Dios.

\begin{tabular}{|c|c|c|}
\hline 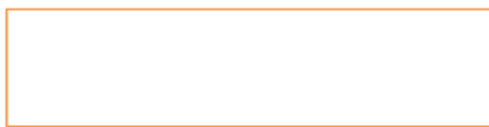 & \multicolumn{2}{|c|}{$\begin{array}{c}\text { Total de } \\
\text { Pacientes } \\
\text { (n: }: 92) \\
\%\end{array}$} \\
\hline EDAD & \multicolumn{2}{|c|}{$\begin{array}{c}63,67 \pm \\
14,06\end{array}$} \\
\hline FALLECIDO & 13 & 14.1 \\
\hline \multicolumn{3}{|l|}{ SEXO } \\
\hline FEMENINO & 51 & 55.4 \\
\hline MASCULINO & 41 & 44.6 \\
\hline TABAQUISMO & 29 & 31.5 \\
\hline TVP & 3 & 3.3 \\
\hline INFECCION & 13 & 14.1 \\
\hline CIRUGIA & 28 & 30.4 \\
\hline CIRUGIA ABDOMINAL & 27 & 29.3 \\
\hline TRANSFUSION (GRE) & 2 & 2.2 \\
\hline EPOC (A) & 1 & 1.1 \\
\hline HEMATOLOGICO & 1 & 1.1 \\
\hline ANGIOTAC & 2 & 2.2 \\
\hline GAMMA V/Q & 0 & 0 \\
\hline \multicolumn{3}{|l|}{ DEFECTO } \\
\hline PROXIMAL & 2 & 2.2 \\
\hline SEGMENTARIO & 1 & 1.1 \\
\hline SUBSEGMENTARIO & 0 & 0 \\
\hline PERIFERICO & 0 & 0 \\
\hline UNILATERAL & 1 & 1.1 \\
\hline BILATERAL & 1 & 1.1 \\
\hline HIPOTENSION & 1 & 1.1 \\
\hline TEP & 2 & 2.2 \\
\hline BAJO RIESGO & 1 & 1.1 \\
\hline SUBMASIVO & 0 & 0 \\
\hline MASIVO & 1 & 1.1 \\
\hline
\end{tabular}

FUENTE: Servicio de Archivo, Hospital San Juan do Dios, C.C.S.S.

\section{CUADRO 2. FRECUENCIA DE PACIENTES CON CANCER DE PANCREAS EN EL PERIODO DE ENERO 2009 A DICIEMBRE 2013 EN EL HOSPITAL SAN JUAN DE DIOS SEGUN CONDICION DE EGRESO}

$\because$ VIVO $\square$ FALLECIDO

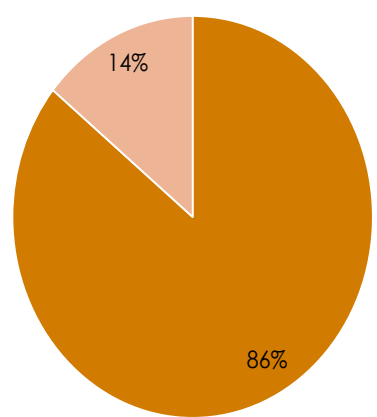

FUENTE: Servicio de Archivo, Hospital San Juan de Dios, C.C.S.S.

Se documentó un número de 2 pacientes con TEP (2.2\%) diagnosticados con CTPA, con defectos de perfusión proximales $(2.2 \%)$, segmentarios $(1.1 \%)$ y bilaterales $(1.1 \%)$ y en uno de lo casos se documentó hipotensión como variable para definir el TEP como masivo (1.1\%) (Tabla I).

\section{DISCUSIÓN}

Al realizar una descripción de la frecuencia de TEP en una población con el diagnóstico de cáncer de páncreas se debe considerar aspectos epidemiológicos y de la interacción de los factores de riesgo para TEP y la neoplasia específica, en un grupo de pacientes cuya incidencia de TEP puede ser menor dado que se analizó los datos de pacientes hospitalizados ${ }^{(10)}$.

La incidencia de cáncer de páncreas a nivel mundial se ha documentado en rangos de 1 a 10 casos por 100.000 pacientes, con una incidencia mayor en países desarrollados, que corresponde a 1.200 casos nuevos por año en países con población de 8 millones de habitantes como Austria ${ }^{(28,29)}$. El incremento en la incidencia de este tipo de neoplasia ha sido reportado en $0.9 \%$ por año en hombres de raza blanca y $1 \%$ por año en mujeres de raza blanca en el periodo de 1999 a 2008 en una cohorte norteamericana ${ }^{(30)}$. De este manera, la incidencia del TEP en este tipo de tumor debe de evaluarse considerando una incidencia esperada de 102 casos de TEP por cada 1000 personas/año ${ }^{(27)}$. 
La edad de los pacientes es un factor a considerar en el análisis de los resultados, dado que la incidencia del cáncer de páncreas tiene un pico entre los 60 y 80 años, con una media en 71 años, siendo raros los casos en pacientes menores de 40 años (2 casos por millón por año) y con una incidencia que se aumenta a 200 casos por 100.000 pacientes año después de los 80 $\operatorname{años}^{(28,29)}$.

La edad media de la población estudiada se encuentra dentro del rango de incidencia descrito; sin embargo, se documentó valores extremos de 49 años con el diagnóstico de este tumor, los cuales podrían tener otros factores de riesgo no evaluados que expliquen la aparición de la neoplasia pancreática a edad más temprana, entre los cuales se puede señalar la presencia de síndromes genéticos descritos con riesgo de $50 \%$ como la Pancreatitis Hereditaria (genes PRSS1, SPINKK1), el Síndrome de Peutz-Jeghers (gen STK11) con $30-40 \%$ de riesgo o el Síndrome de Lynch (genes MLH1, MSH2, MSH6) ${ }^{(28)}$.

La mortalidad en el paciente con cáncer de páncreas evaluada en este estudio puede estar sesgada por la inclusión exclusiva de pacientes hospitalizados, de manera que el $14.1 \%$ de pacientes fallecidos reportado está sujeto al diagnóstico intrahospitalario del fallecimiento. En todo caso, la presencia de un TEP en el paciente con neoplasia pancreática predice un aumento de mortalidad prematura ( 3 meses) que debe evaluarse en el contexto de la mortalidad global por este cáncer reportada en tasas de 15.8 por millón de pacientes en hombres y 12.6 por millón de pacientes en mujeres ${ }^{(15,16)}$.

La razón de incidencia entre hombres y mujeres con el diagnóstico de cáncer de páncreas se ha reportado con valores aproximados de 1:1.5 y difiere con lo observado en este estudio dado que se evidenció una relación de 1.24 con una mayor frecuencia en mujeres (Tabla I) ${ }^{(29)}$. Esta diferencia debe de analizarse en conjunto con otros factores de riesgo, el tamaño de la población estudiada y el diseño del estudio para establecer una asociación definitiva.

Dentro de los factores de riesgo identificados como posibles contribuyentes o variables confusoras en el análisis de la frecuencia de TEP en este grupo de pacientes con cáncer de páncreas, se identificó el tabaquismo, la cirugía abdominal y la presencia de infección. Tanto la cirugía abdominal como la presencia de infección se han establecido como factores de riesgo para TEP en pacientes independientemente de la presencia de una neoplasia ${ }^{(17,23,24,31-34)}$.

El tabaquismo presenta un hazard ratio (HR) de 1.52 para tromboembolismo pulmonar asociado o no a cáncer ${ }^{(35)}$. En el caso del paciente con cáncer de páncreas, este riesgo debe de analizarse contemplando que el paciente con tabaquismo aumenta 2-3 veces el riesgo de cáncer de páncreas, con un estimado de $25 \%$ de este tipo de neoplasias asociadas a la exposición al tabaco y con un riesgo proporcional al número de paquetes año fumados ${ }^{(28,29)}$.

La presencia de un antecedente de tabaquismo en $31.8 \%$ de los pacientes podría aumentar el riesgo de ambos eventos, TEP y cáncer de páncreas, de modo que el diseño del estudio no permite establecer una asociación entre estas dos variables. Por otro lado, la presencia de un porcentaje alto de tabaquismo se ha asociado a una presentación temprana (10 años) del cáncer de páncreas, un factor que podría explicar los extremos de edad al diagnóstico del tumor mencionados previamente ${ }^{(29)}$.

Los defectos de perfusión documentados por la tomografía computarizada con angiografía espiral (CTPA) en los pacientes con diagnóstico de TEP en este estudio correlacionan con la sensibilidad moderada-alta para lesiones proximales de esta herramienta diagnóstica ${ }^{(36-38)}$.

\section{CONCLUSIONES Y RECOMENDACIONES}

En los pacientes hospitalizados con diagnóstico de cáncer de páncreas en el periodo de enero 2009 a diciembre 2013 en el Hospital San Juan de Dios se documentó un 2,2\% de TEP.

Los factores de riesgo para TEP más frecuentes en los pacientes hospitalizados con diagnóstico de cáncer de páncreas en el periodo de enero 2009 a diciembre 2013 en el Hospital San Juan de Dios fueron el tabaquismo, la cirugía abdominal y la presencia de infección.

El diseño del estudio de investigación no permite establecer asociaciones de riesgo entre las variables estudiadas como factores de riesgo para TEP en la población analizada. Además, dada esta 
situación no se puede evaluar la presencia de variables confusoras como el tabaquismo.

Con los resultados obtenidos en este trabajo de investigación es necesario plantear un estudio de investigación de cohorte con una población control para definir asociaciones de riesgo para TEP en los pacientes con cáncer de páncreas de la población costarricense

\section{REFERENCIAS BIBLIOGRÁFICAS}

1. Rosendaal FR. Venous thrombosis: a multicausal disease. Lancet. 1999;353:11671173.

2. Trousseau A. Phlegmasia Alba Dolens: Lectures on Clinical Medicine. London, England: The New Sydenham Society; 1868:5:281-331.

3. Tapson V. Acute pulmonary embolism. N Engl J Med. 2008;358:1037-1052.

4. Silverstein MD Heit JA Mohr DN et al. Trends in the incidence of deep vein thrombosis and pulmonary embolism: a 25-year population-based study. Arch Intern Med. 1998;158:585-593.

5. Heit JA Cohen AT Anderson FA. VTE Impact Assessment Group. Estimated annual number of incident and recurrent, non-fatal and fatal venous thromboembolism (VTE) events in the U.S. Blood 2005; 106:267a. abstract.

6. Hoffmann B Gross CR Jöckel KH Kröger K. Trends in mortality of pulmonary embolism an international comparison. Thromb Res. 2010;125(4):303-308.

7. Kyrle PA Eichinger S. Deep vein thrombosis. Lancet. 2005;365(9465):1163-1174.

8. Cushman M Tsai AW White RH et al. Deep vein thrombosis and pulmonary embolism in two cohorts: the longitudinal investigation of thromboembolism etiology. Am J Med 2004;117:19-25.

9. Killewich LA Bedford GR Beach KW Strandness D. Spontaneous lysis of deep venous thrombi: rate and outcome. J Vasc Surg. 1989;9:89-97.

10. Heit JA Melton LJ 3rd Lohse CM et al. Incidence of venous thromboembolism in hospitalized patients vs community residents. Mayo Clin Proc. 2001;76(11):11021110 .

11. Oger E. Incidence of venous thromboembolism: a community-based study in Western
France. EPI-GETBP Study Group. Groupe d'Etude de la Thrombose de Bretagne Occidentale. Thromb Haemost. 2000;83(5):657660.

12. Molina JA Jiang ZG Heng BH Ong BK. Venous thromboembolism at the National Healthcare Group, Singapore. Ann Acad Med Singapore. 2009;38(6):470-478.

13. Stein PD Beemath A Olson RE. Trends in the incidence of pulmonary embolism and deep venous thrombosis in hospitalized patients. Am J Cardiol. 2005;95(12):15251526.

14. Yang Y Liang L Zhai Z et al. Pulmonary embolism incidence and fatality trends in Chinese hospitals from 1997 to 2008: a multicenter registration study. PLoS One. 2011;6(11):e26861.

15. Habib M Wasif Saif M. Thromboembolism and Anticoagulation in Pancreatic Cancer. JOP. J Pancreas (Online). 2013;14(2):135137.

16. Phillips A Jacobson J Magai C et al. Cancer Incidence and Mortality in the Caribbean. Cancer Investigation. 2007;25:476-483.

17. Sansone JM del Rio AM Anderson PA. The prevalence of and specific risk factors for venous thromboembolic disease following elective spine surgery. Joint Surg Am. 2010;92(2):304-313.

18. Kim SC Schneeweiss S Liu J Solomon DH. Risk of venous thromboembolism in patients with rheumatoid arthritis. Arthritis Care Res (Hoboken). 2013;65(10):1600-1607.

19. Bacani AK Gabriel SE Crowson CS Heit JA Matteson EL. Non-cardiac vascular disease in rheumatoid arthritis: increase in venous thromboembolic events? Arthritis Rheum. 2012;64(1):53-61.

20. Smeeth L Cook C Thomas S Hall AJ Hubbard R Vallance P. Risk of deep vein thrombosis and pulmonary embolism after acute infection in a community setting. Lancet. 2006;367(9516):1075-1079.

21. Neumann V O'Connor RJ Bhakta BB Tennant A. DVT and pulmonary embolism after acute infection. Lancet. 2006;368(9531): 201.

22. Bahl V Hu HM Henke PK Wakefield TW Campbell DA Jr Caprini JA. A validation study of a retrospective venous thromboembolism risk scoring method. Ann Surg. 2010;251(2):344-350

23. Khorana AA Francis $\mathrm{CW}$ Blumberg $\mathrm{N} \mathrm{Cu}-$ lakova E Refaai MA Lyman GH. Blood 
transfusions, thrombosis, and mortality in hospitalized patients with cancer. Arch Intern Med. 2008;168(21):2377-2381.

24. Holst AG Jensen G Prescott E. Risk factors for venous thromboembolism: results from the Copenhagen City Heart Study. Circulation. 2010;121(17):1896-1903.

25. Baron JA Gridley G Weiderpass E Nyren O Linet M. Venous thromboembolism and cancer. Lancet. 1998;351:1077-1080.

26. Horsted F West J Grainge MJ. Risk of venous thromboembolism in patients with cancer: a systematic review and meta-analysis. "Risk of Venous Thromboembolism in Patients with Cancer”. PLoS Medicine 9.7 (2012): e1001275.

27. Prandoni P Lensing AW Cogo A et al. The long-term clinical course of acute deep venous thrombosis. Ann Intern Med. 1996; 125:1-7.

28. Ryan D Hong T Bardeesy N. Pancreatic Adenocarcinoma. N Engl J Med 2014;371: 1039-1049.

29. Krejs G. Pancreatic Cancer: Epidemiology and Risk Factors. Dig Dis. 2010;28:355358 .

30. Simard E Ward E Siegel R Jemal A. Cancers With Increasing Incidence Trends in the United States: 1999 Through 2008. CA Cancer J Clin. 2012;62:118-128.

31. Cohen AT. Prevention of postoperative venous thromboembolism. BMJ. 2009;339: b4477.

32. Cheng JS Arnold PM Anderson PA Fischer D Dettori JR. Anticoagulation risk in spine surgery. Spine (Phila Pa 1976). 2010;35(9 Suppl):S117-S124.

33. Hansson PO Eriksson H Welin L Svärdsudd $\mathrm{K}$ Wilhelmsen L. Smoking and abdominal obesity: risk factors for venous thromboembolism among middle-aged men: "the study of men born in 1913”. Arch Intern Med. 1999;159(16):1886-1890.

34. Douketis J Tosetto A Marcucci M et al. Risk of recurrence after venous thromboembolism in men and women: patient level metaanalysis. BMJ. 2011;342:d813.

35. Ray JG Burows RF Ginsberg JS Burrows EA. Paroxysmal nocturnal hemoglobinuria and the risk of venous thrombosis: review and recommendations for management of the pregnant and nonpregnant patient. Haemostasis. 2000;30(3):103-117.

36. Kelly MJ Pitman A. Defining the role of helical computerised tomography (CT) in the diagnosis of pulmonary embolism (PE). Aust N Z J Med. 2000;30(2):191-193.

37. Stein PD Fowler SE Goodman LR et al. Multidetector computed tomography for acute pulmonary embolism. N Engl J Med. 2006;354(22):2317-2327.

38. Brotman DJ. Computed tomography for pulmonary embolism. N Engl J Med. 2006; 355(9):955.

\section{FUENTES DE APOYO}

No hay fuentes de apoyo que declarar.

\section{CONFLICTO DE INTERÉS}

No hay conflicto de interés que declarar. 